\title{
Isoniazid-induced cerebellitis: a disguised presentation
}

\author{
Prasant $\underline{\text { Peter }}^{1}, \mathrm{MD}$, Mary $\underline{\text { John }^{2}}, \mathrm{MD}$
}

\begin{abstract}
Cerebellitis is a rarely encountered complication of isoniazid therapy. Its occurrence is usually associated with concomitant renal disease and haemodialysis. Herein, we report the case of a patient with this complication who presented with isolated bilateral symmetrical dentate nucleus T2 hyperintensities on magnetic resonance imaging. Isoniazid neurotoxicity has never been reported to cause bilateral dentate hyperintensities, for which the differentials are few and include metronidazole toxicity.
\end{abstract}

Keywords: bilateral dentate nuclei, cerebellitis, chronic kidney disease, isoniazid

\section{INTRODUCTION}

Chronic kidney disease (CKD) and tuberculosis have been proven to be interrelated. An increased incidence of tuberculosis, as well as side effects of antituberculosis therapy, has been reported in patients with $C K D$, especially in those on haemodialysis. ${ }^{(1)}$ Although the neurotoxic complications of isoniazid $(\mathrm{INH})$ are well known, cerebellitis is rarely reported as one of them. .1-3) $^{-}$

Our patient was a middle-aged woman who presented with vertigo following the initiation of antituberculosis therapy. On investigation, the patient was diagnosed to have CKD, and INH neurotoxic complications were diagnosed through a process of exclusion. Dramatic improvement was observed with the discontinuation of INH and supplementation with highdose pyridoxine. Magnetic resonance (MR) imaging findings of isolated bilateral dentate nucleus signal alteration were unusual, as INH neurotoxicity is generally not included in the differential diagnosis of this presentation.

\section{CASE REPORT}

A 40-year-old Asian woman presented to the outpatient department of the Christian Medical College and Hospital, Ludhiana, India, with complaints of dizziness and slurring of speech for the past one week. She reported that she experienced episodes of dizziness on sitting and walking, but was asymptomatic lying down. She had no history of headaches, weakness, nausea or vomiting. The patient appeared distressed during examination. On abdominal palpation, mild pyrexia and hepatosplenomegaly were observed. Examination of the central nervous system revealed bilateral cerebellar signs. Two weeks prior to her presentation, the patient had been started on antituberculosis therapy (directly observed therapy short course category 1) following a diagnosis of pulmonary tuberculosis, which was made based on a positive polymerase chain reaction test from the bone marrow.

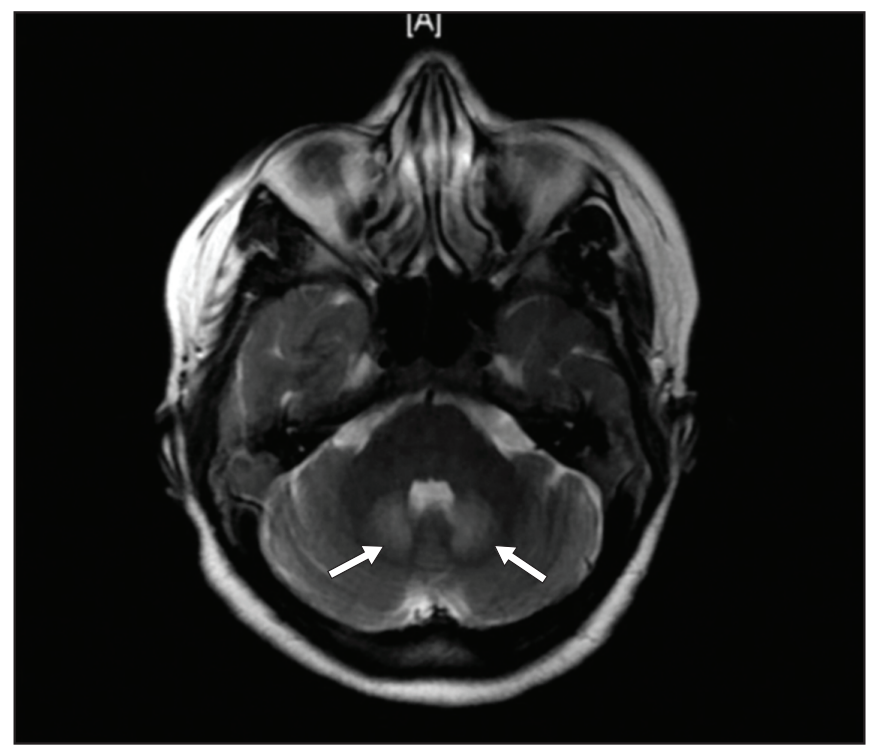

Fig. 1 Axial T2-W MR image shows symmetrical dentate nuclei hyperintensities (arrows).

Investigations revealed a raised erythrocyte sedimentation rate $(110 \mathrm{~mm} / \mathrm{hr})$, deranged liver function tests (alanine transaminase at 4,000 IU/L, lactate dehydrogenase at $563 \mathrm{IU} / \mathrm{L}$ ), increased blood urea $(96 \mathrm{mg} / \mathrm{dL}$ ) and an increased creatinine level $(2.3 \mathrm{mg} / \mathrm{dL})$. Chest radiography showed opacity in the left lower zone, and ultrasonography of the abdomen showed bilateral echogenic kidneys with loss of corticomedullary differentiation, which is suggestive of chronic renal parenchymal disease. Other investigations, including cerebrospinal fluid (CSF) analysis, were normal. In view of the repeated attacks of dizziness, the patient underwent MR imaging of the brain, which revealed bilateral symmetrical dentate nucleus hyperintensities on T2weighted (Fig. 1) and T2 fluid-attenuated inversion recovery (FLAIR) images (Fig. 2). The dentate nuclei appeared isointense on T1-weighted images and showed no evidence of restricted diffusion on diffusion-weighted imaging (Fig. 3). No other areas of signal alteration were noted in the bilateral cerebral or

${ }^{1}$ Department of Radiodiagnosis, ${ }^{2}$ Department of Internal Medicine, Christian Medical College and Hospital, Ludhiana, Punjab, India

Correspondence: Dr Prasant Peter, Assistant Professor, Department of Radiodiagnosis, Christian Medical College and Hospital, Ludhiana, Punjab, Pin-141008, India. prasant.peter@rediffmail.com 


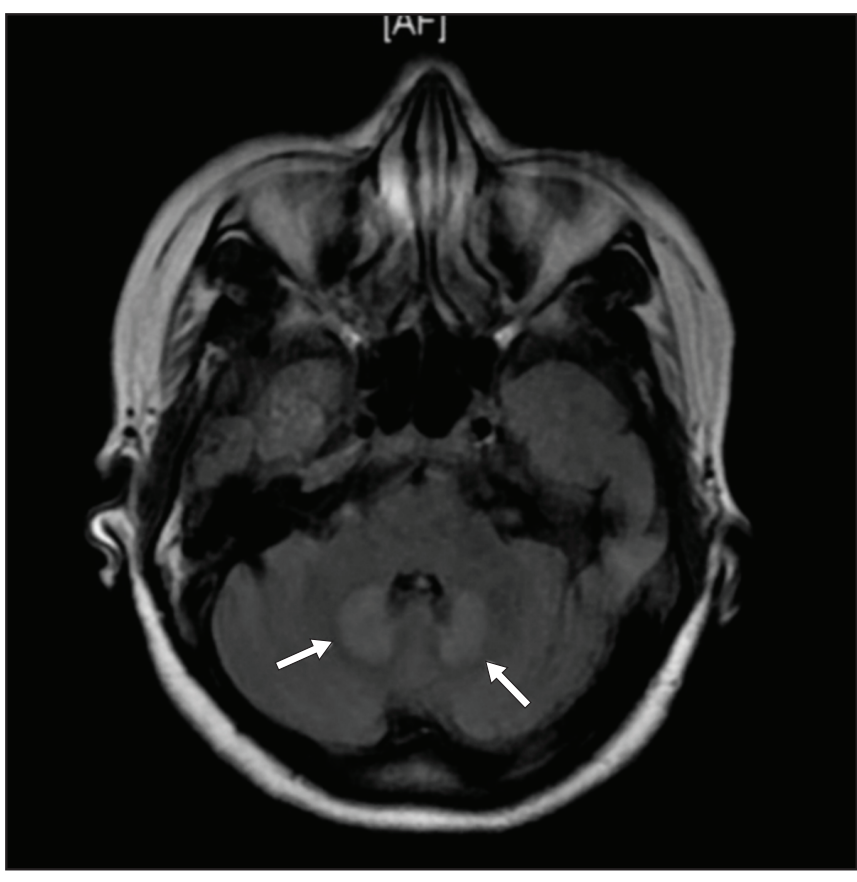

Fig. 2 Axial T2 FLAIR MR image shows symmetrical hyperintensities involving the bilateral dentate nuclei (arrows).

cerebellar hemispheres. Based on the patient's clinical history, physical examination and CSF analysis, the reported causes of bilateral symmetrical dentate nucleus hyperintensities, including enteroviral encephalitis and metronidazole toxicity, were excluded. Cerebellitis due to INH neurotoxicity secondary to reduced renal clearance was then considered as a possible aetiology. Accordingly, INH administration was discontinued and the patient was started on high-dose pyridoxine $(100 \mathrm{mg} /$ day). The patient experienced dramatic improvement in her symptoms, further confirming the diagnosis. She was subsequently discharged on daily pyridoxine supplementation, and a followup session was scheduled one month later. At follow-up, the patient remained asymptomatic. Repeat MR imaging of the brain six weeks post discharge showed complete resolution of the bilaleral dentate nucleus hyperintensities (Fig. 4).

\section{DISCUSSION}

A diagnosis of drug toxicity is made primarily through a process of exclusion. To make such a diagnosis, it is important to demonstrate a close association between the onset of drug usage and the onset of symptoms, as was evidenced in our patient. Other than the close association observed, our patient also demonstrated dramatic improvement in her symptoms as soon as the drug was discontinued and pyridoxine therapy initiated. This further strengthened the diagnosis of druginduced cerebellitis. Other causes of cerebellitis, such as excessive alcohol intake, use of other medications, stroke, vaccinations and infective aetiologies, were ruled out through careful history taking, and clinical examination and investigations, including CSF analysis. Follow-up MR imaging at six weeks post discharge likewise showed resolution of the signal alterations.

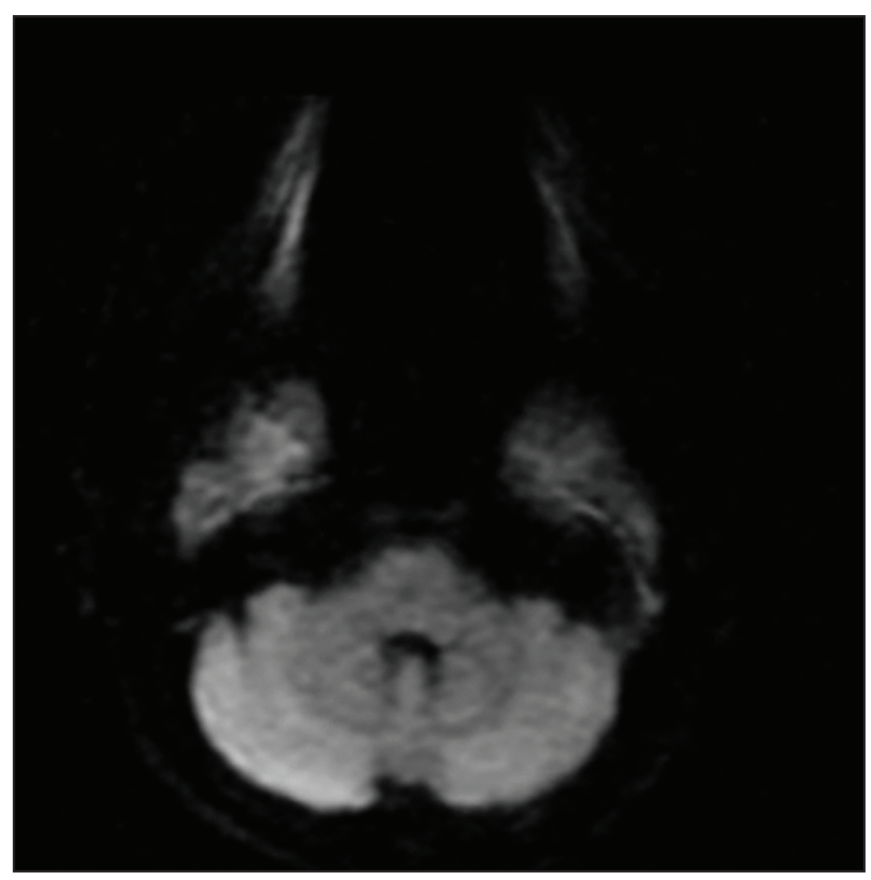

Fig. 3 Diffusion-weighted MR image shows no evidence of restricted diffusion in the dentate nuclei.

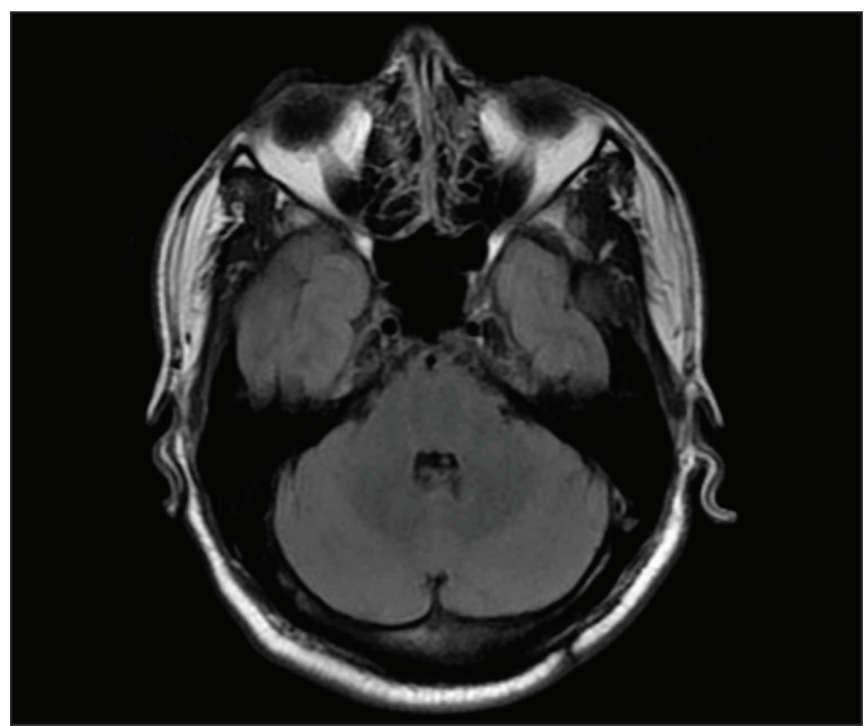

Fig. 4 Axial T2 FLAIR MR image shows complete resolution of the altered signal intensity in the bilateral dentate nuclei at six weeks post discharge.

On investigation, our patient was diagnosed with CKD, a condition that would have reduced the clearance of INH (which has a primarily renal route of excretion) and contributed to higher levels of $\mathrm{INH}$, leading to neurotoxicity. The neurotoxic effects of INH are reported to be due to the inhibition of pyridoxine phosphorylation, ${ }^{(1)}$ and therefore, pyridoxine supplementation is advised. However, even with pyridoxine supplementation (15-50 mg/day), up to $5 \%$ of patients would still have some adverse effects, with the proportion rising fourfold when pyridoxine dosage is inadequate. ${ }^{(3)}$ The recommended dosage of pyridoxine for the prevention of INH toxicity in patients on haemodialysis is as high as $100 \mathrm{mg} /$ day $^{(4)}$ 
Another particularly interesting aspect of our patient was the finding of isolated bilateral symmetrical dentate nucleus hyperintensities on T2-weighted and T2 FLAIR MR imaging. As acute cerebellitis usually presents with diffuse signal alterations throughout the bilateral cerebellar hemispheres, ${ }^{(5)}$ the isolated involvement of the dentate nuclei in our patient was unusual. Only few pathologies are included in the differential diagnoses of bilateral dentate nuclei hyperintensities on T2-weighted MR images. These include methyl bromide intoxication, enteroviral encephalopathies, maple syrup urine disease and metronidazole toxicity. ${ }^{(6,7)}$ Bilateral dentate involvement has also been described as part of the atypical presentation of Wernicke encephalopathy, ${ }^{(8)}$ although not in isolation. In the present case, all these differentials were ruled out based on the patient's medical history and the results of clinical investigations (including CSF analysis). Therefore, by a process of exclusion, we concluded that the unusual imaging presentations seen in our patient were a result of INH neurotoxicity.

In conclusion, the possibility of cerebellitis secondary to INH neurotoxicity should be considered in patients with CKD who present with neurological complaints after starting antituberculosis therapy. As a preventive strategy, treatment with high-dose pyridoxine should be initiated for patients with known CKD who are being started on antituberculosis therapy. INH should also be withheld if symptoms of neurotoxicity develop. INH-induced neurotoxicity should be included in the differential diagnosis of bilateral symmetrical dentate nucleus hyperintensities on T2-weighted or T2 FLAIR MR images.

\section{REFERENCES}

1. Tan M, Alkan O, Karaca S, Karakurum Goksel B, Demiroglu YZ. Cerebellitis due to Antituberculosis Therapy in a Patient with Chronic Renal Failure. Fırat Tıp Dergisi 2009; 14:290-2.

2. Blumberg EA, Gil RA. Cerebellar syndrome caused by isoniazid. DICP 1990; 24:829-31.

3. Lewin PK, McGreal D. Isoniazid toxicity with cerebellar ataxia in a child. CMAJ 1993; 148:49-50.

4. Siskind MS, Thienemann D, Kirlin L. Isoniazid-induced neurotoxicity in chronic dialysis patients: report of three cases and a review of literature. Nephron 1993; 64:303-6.

5. De Bruecker $Y$, Claus F, Demaerel $P$, et al. MRI findings in acute cerebellitis. Eur Radiol 2004; 14:1478-83.

6. Kim E, Na DG, Kim EY, et al. MR imaging of metronidazole-induced encephalopathy: lesion distribution and diffusion-weighted imaging findings. AJNR Am J Neuroradiol 2007; 28:1652-8.

7. Kalia V, Vibhuti, Saggar K. Case report: MRI of the brain in metronidazole toxicity. Indian J Radiol Imaging 2010; 20:195-7.

8. Kang SY, Kang JH, Choi JC, Choi G. Wernicke's encephalopathy: unusual manifestation on MRI. J Neurol 2005; 252:1550-2. 